\title{
Responding to COVID-19: The UW Medicine Information Technology Services Experience
}

\author{
Elisha S. Grange ${ }^{1}$ Eric J. Neil ${ }^{1} \quad$ Michelle Stoffel $^{2,3}$ Angad P. Singh ${ }^{1,4}$ Ethan Tseng ${ }^{3,5}$

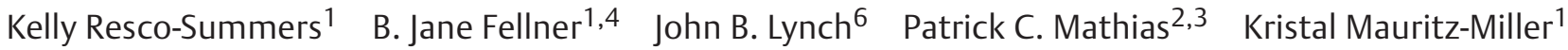 \\ Paul R. Sutton ${ }^{1,7}$ Michael G. Leu ${ }^{1,3,8,9}$
}

\footnotetext{
${ }^{1}$ Department of Information Technology Services, UW Medicine, Seattle, Washington, United States

${ }^{2}$ Department of Laboratory Medicine, University of Washington, Seattle, Washington, United States

${ }^{3}$ Department of Biomedical Informatics and Medical Education, University of Washington, Seattle, Washington, United States

${ }^{4}$ Department of Family Medicine, University of Washington, Seattle, Washington, United States

${ }^{5}$ Department of Emergency Medicine, University of Washington, Seattle, Washington, United States

6 Division of Allergy and Infectious Diseases, Department of Medicine, University of Washington, Seattle, Washington, United States

${ }^{7}$ Division of General Internal Medicine, Department of Medicine, University of Washington, Seattle, Washington, United States

8 Department of Pediatrics, University of Washington, Seattle, Washington, United States

${ }^{9}$ Seattle Children's Hospital, Seattle, Washington, United States
}

\begin{abstract}
Address for correspondence Elisha S. Grange, MSS, MDIL, Strategic Communications, UW Medicine IT Services, 325 9th Avenue, Seattle, WA 98104-2499, United States (e-mail: egrange@uw.edu).
\end{abstract}

Appl Clin Inform 2020;11:265-275.

\section{Abstract}

Keywords

- COVID-19

- health information technology

- public health
Background UW Medicine was one of the first health systems to encounter and treat COVID-19 patients in the United States, starting in late February 2020.

Objective Here we describe the rapid rollout of capabilities by UW Medicine Information Technology Services (ITS) to support our clinical response to the COVID19 pandemic and provide recommendations for health systems to urgently consider, as they plan their own response to this and potentially other future pandemics.

Methods Our recommendations include establishing a hospital incident command structure that includes tight integration with IT, creating automated dashboards for incident command, optimizing emergency communication to staff and patients, and preparing human resources, security, other policies, and equipment to support the transition of all nonessential staff to telework.

We describe how UW Medicine quickly expanded telemedicine capabilities to include most primary care providers and increasing numbers of specialty providers. We look at how we managed expedited change control processes to quickly update electronic health records (EHR) with new COVID-19 laboratory and clinical workflows. We also examine the integration of new technology such as tele-intensive care (ICU) equipment and improved integration with teleconferencing software into our EHR. To support the rapid preparation for COVID-19 at other health systems, we include samples of the UW Medicine's COVID-19 order set, COVID-19 documentation received

March 25, 2020

accepted after revision

March 29, 2020
DOI https://doi.org/

10.1055/s-0040-1709715.

ISSN 1869-0327. (c) 2020 Georg Thieme Verlag KG

Stuttgart · New York

\section{License terms \\ (이 (1) $\Theta \circledast$}


template, dashboard metric categories, and a list of the top 10 things your health care IT organization can do now to prepare.

Conclusion The COVID-19 response requires new and expedited ways of approaching ITS support to clinical needs. UW Medicine ITS leadership hope that by quickly sharing our nimble response to clinical and operational requests, we can help other systems prepare to respond to this public health emergency.

\section{Background and Significance}

The novel coronavirus (COVID-19), which originated in late 2019, presents an unprecedented challenge to health systems. The potential for asymptomatic rapid spread and the high mortality rate for vulnerable elderly and immunocompromised populations make rapid identification and containment of positive patients essential. ${ }^{1}$ Within the United States, the first COVID-19 patient was identified in Washington State in January, $2020^{2}$ and Washington was the early epicenter of the U.S. outbreak. ${ }^{3}$

UW Medicine is a large health care organization in the Pacific Northwest with nearly 30,000 personnel. Our organization includes four hospitals, 15 neighborhood clinics, the University of Washington School of Medicine, an air ambulance service, and a wide network of practitioners. Combined, UW Medicine has 64,220 hospital admissions, 1,675,442 clinic visits, 204,634 emergency department visits each year, and 1,544 licensed beds. Information technology services (ITS) consist of 528 employees responsible for the IT infrastructure and clinical applications of UW Medicine.

UW Medicine acted quickly to establish structures and systems to respond to COVID-19. A task force was established on February 29, 2020, to monitor COVID-19 issues. We created a new governance to enable a more rapid response to COVID-19 issues, as they arose and focused on ways that IT could support the ongoing clinical needs of the organization. Through this governance, we created new communication systems, accelerated efforts to support our workforce, and worked to identify and mitigate new security concerns. In this manuscript, we seek to characterize the health information technology challenges we faced, to share our newly acquired knowledge more broadly with the informatics community, and to facilitate the rapid adaptation of other health systems to the public health threat of COVID-19 and other potential future emergencies of this type.

\section{New Structures to Accelerate Response}

\section{Hospital Incident Command and ITS Emergency Management}

Hospital incident command is an approach to systemic organization during exceptional events. ${ }^{4,5}$ UW Medicine utilizes an enterprise-wide Hospital Incident Command System (HICS) approach to manage major incidents. Eight site-based incident command centers (SICCs) are established and are linked to the enterprise HICS through shared planning, logistics, and operational groups (-Fig. 1). The enter- prise HICS addresses system-wide concerns and provides overall coordination. UW HICS partnered with King County Public Health, Washington State Department of Health, the Northwest Healthcare Response Network, the U.S. Centers for Disease Control and Prevention, and other local, regional, and national organizations.

IT integration into the HICS is managed via the ITS Business Continuity and Disaster Recovery program, which coordinates and leads our ITS emergency management activities. The IT incident commander (IT IC) represents ITS and reports to the HICS logistics' chief. Both the ITS chief information officer and IT IC participated in twice daily phone briefings with HICS. The IT IC cascades information to the ITS executive team through a daily 07:00 meeting of ITS executives concerning COVID-19, where the IT IC shares guidance from the HICS and gathers feedback to report back up. The IT IC also disseminates an e-mail update to executives every other day. The ITS workforce receives updates on a daily to twice weekly e-mail schedule.

ITS clinical informaticians are seated in individual SICCs; and the IC coordinates, three IT focus threads are people, electronic health record (EHR)/clinical, and technology; each of which has an internal IT leader responsible for assigning resources and tracking work to completion. As the pandemic progressed and demand for ITS increased to round-the-clock, additional IT program managers (PM) were reassigned to support the IT IC with COVID-19. For example, one PM was put in charge of COVID-19 project intake to help clarify incoming requests for equipment and new capabilities.

Enterprise and site-based command center staff were expected to be available during core hours of 07:30 to 16:30, with tasks completed by personnel working through the night if needed. We rotated responsibility for the key position of IT IC, enabling ITS to switch from sprints to marathon support. This is very different than historical disaster-related responses that are usually linked to short-lived span, though highly impactful events such as mass shootings and earthquakes.

\section{New Governance to Accelerate Response}

The ability to adapt quickly while maintaining system stability is critical. ITS typically uses a mixture of methodologies that include waterfall and elements of iterative development. The COVID-19 ITS response included many small projects and some much bigger. We are moving to more iterative implementations in some areas that would have been pure waterfall otherwise. For example, COVID-19 related change requests to the EHR were automatically classified as emergency change requests. The change control process for larger, more complex 


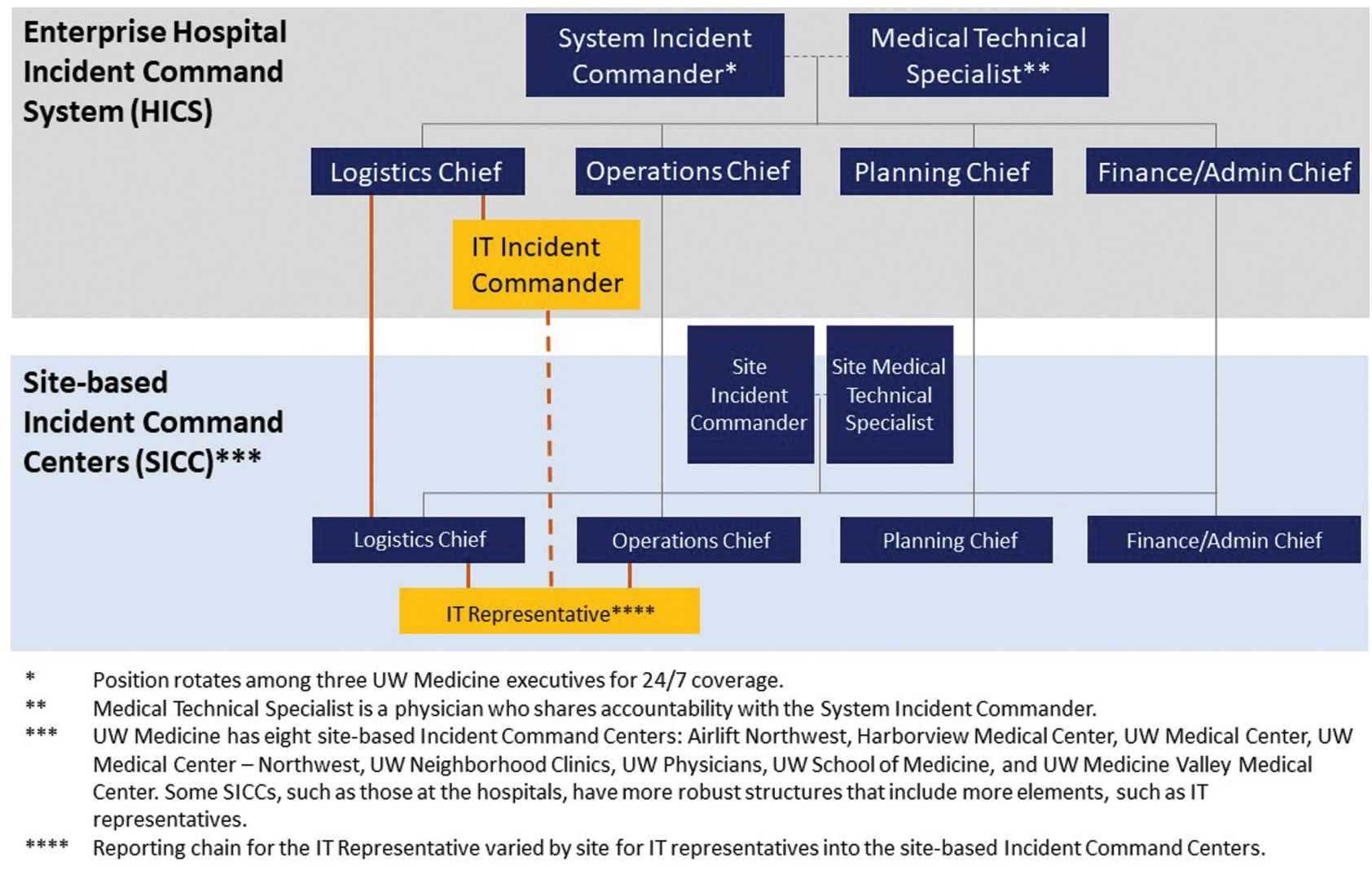

Fig. 1 IT Services integration into hospital incident command system structure.

changes is usually on the order of weeks. We developed a modified change control process for COVID-19-related items. Changes were evaluated and implemented within hours, while still maintaining internal change control and consistent IT practices.

Early EHR change requirements included creating an order set for home visits by a newly created home assessment team, ${ }^{6}$ precautions for blood processing, orders, standard documentation phrasing, and alerts. COVID-19 related requests were being made at all hours of the day. Our IT IC allowed us to quickly operationalize new guidance by incorporating it into EHRs. We monitored our EHR vendor sites for emergent system updates and have designated IT employees staffed around the clock to evaluate and implement change requests.

\section{New Supportive Dashboards}

Enterprise IC needed visibility of events, actions, and changes happening across the enterprise to support decision making in the HICS. They provided metric requirements, and ITS created a real-time dashboard (-Fig. 2) of critical metrics including the number of tests by result and facility per day, laboratory turnaround time, current admitted patient counts, counts of personal protective equipment on hand by facility, and telehealth visits per day related to COVID-19. More recently, we tracked patients in intensive care unit (ICU), as well as the number, and percentage of COVID-19 positive patients. We are using this information to project COVID-19-related care demand. A full list of the metrics collected by incident command is collated in - Table 1 .
In addition to the enterprise IC dashboard, we created specialized reports including a dashboard for the laboratory and supply chain, operational reports, and patient lists. Laboratory dashboards identify tested patients, turnaround times, and specimen source location information. These reports are automated where possible, allowing decision makers to analyze data in a self-service manner, and to ensure their continuous availability.

\section{Optimize Communication to Support New Needs}

A rapidly evolving pandemic situation requires real-time internal and external communication. ITS created a variety of push and pull platforms to support the UW Medicine workforce, and our wider community, including patients.

In addition to the executive communications described previously, UW Medicine sought to communicate regular updates to staff across the enterprise. Daily updates were initially sent (1) as e-mails to senior leaders, who were tasked with forwarding as indicated or (2) via "The Huddle," a daily newsletter to all UW Medicine staffs. The lack of centralized communication capability (stemming from the fact that facilities within the enterprise typically manage their own staff communication) resulted in a flood of e-mails that were difficult to process and prioritize by end users. In response, ITS and UW Medicine internal communications collaborated with human resources (HR) and local sites to create more complete distribution lists, including sublists targeting specific stakeholder groups for COVID-19 outreach. As these efforts 


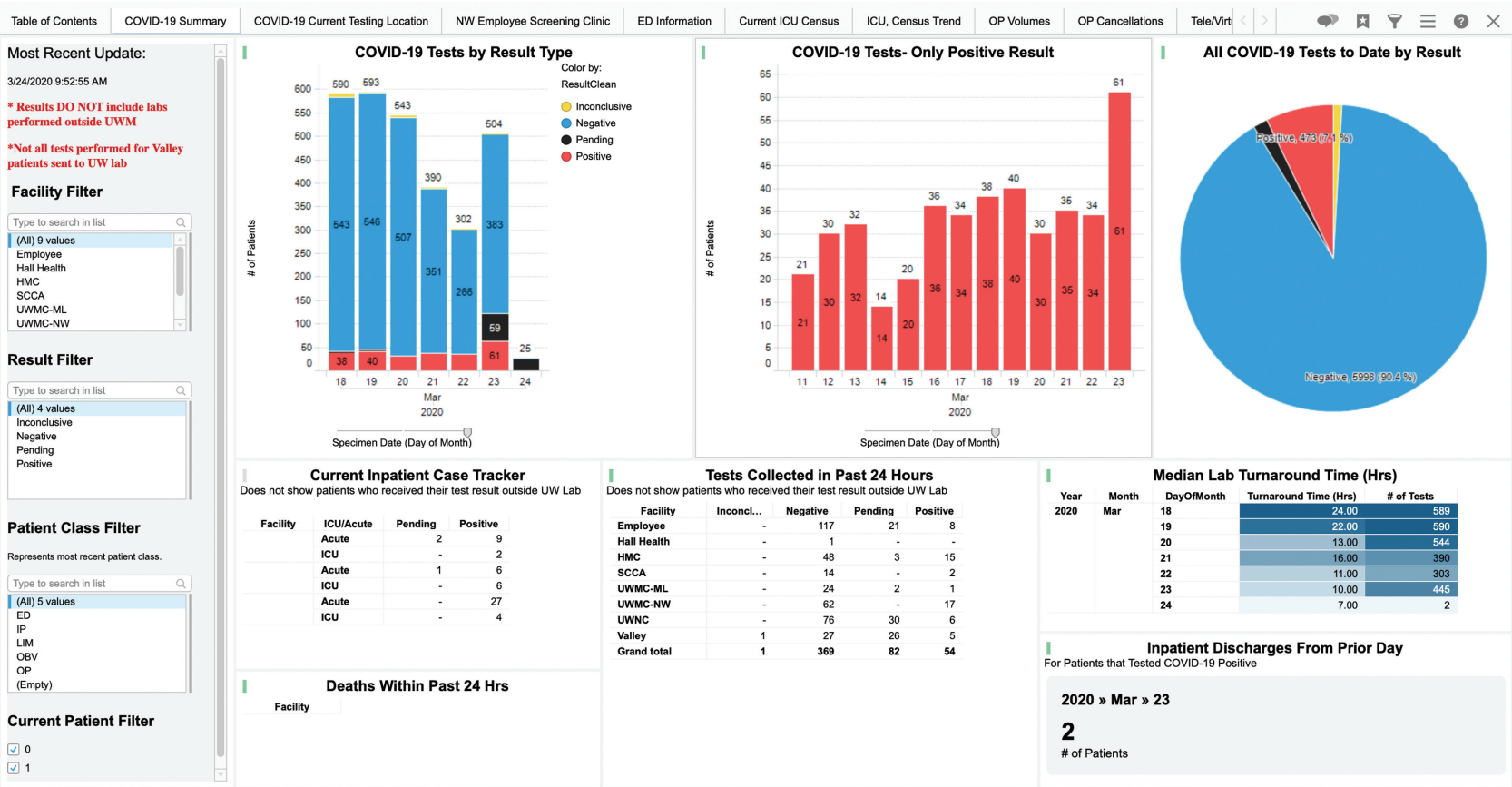

Fig. 2 COVID-19 incident command dashboard. UW Medicine IT services created a dashboard for our COVID-19 hospital incident command. A list of metrics collected can be found in - Table 1.

\section{Table 1 COVID-19 dashboard metrics}

\begin{tabular}{|l|}
\hline The following metrics are collected by UW Medicine COVID-19 Incident Command \\
\hline - No. of tests by result and by facility per day \\
\hline - Laboratory turnaround time \\
\hline - No. of patients currently in ICUs as well as the no. and \% of those patients who are COVID-19 positive \\
\hline - No. of current positive and pending tests among inpatients \\
\hline - Current location of patients with positive or pending tests \\
\hline - On hand supply counts of personal protective equipment at each facility \\
\hline - No. of outpatient visits per day \\
\hline - No. of outpatient no shows per day \\
\hline - No. of outpatient cancellations per day (separated by COVID-19-related or not) \\
\hline - No. of ED visits per day \\
\hline - ED Length of stay \\
\hline - List of test results for patients tested who presented at the ED \\
\hline - Telehealth visits per day for COVID-19-related symptoms \\
\hline - COVID-19 info line calls per day (by nurse triage needed or not) \\
\hline - \% of calls abandoned and average call speed for calls into COVID-19 info line \\
\hline
\end{tabular}

Abbreviations: COVID-19, novel coronavirus; ED, emergency department; ICU, intensive care unit.

progressed, UW Medicine was able to reduce its reliance on cascading e-mails and reduced redundant e-mails. Communications became more focused and tightly organized.

Communicating EHR changes is critical to ensure that clinical staff are aware of the latest COVID-19 tools. ITS sent e-mail concerning special updates to users (overriding previous unsubscribe requests) and repeated news in four enduser newsletters. ITS physicians also sent change information to expert users, and hospital/physician leadership.
Communication to the community is more complex than communicating with staff given the variety of public outreach mechanisms, ranging from multiple UW Medicine web sites, automated patient e-mail and text patient notifications (e.g., appointment reminders), and hold messages when calling the UW Medicine Contact Center. The IT IC worked with the owners of these groups to align external messaging to enterprise COVID-19 updates regarding selfscreening and testing guidance, transition from in-person 
to telehealth visits, and discontinuation of online selfscheduled appointments.

To ensure consistency, ITS created an intranet and an extranet page to house COVID-19 resources. The internal webpage included screening and testing algorithms, policies and procedures, and signs and posters for clinics. The internal webpage clearly describes what content is UW Medicine only, and what can be shared externally. The external webpage (launched on March 11, 2020), shared COVID-19 resources with others. ${ }^{7}$

ITS also helped the organization to stay connected by increasing staff at the Help Desk and preparing them to answer questions and quickly resolve issues from clinicians using new telehealth capabilities, and employees are now working remotely.

\section{Supporting Medical Practice}

UW Medicine targeted rapid laboratory testing and result notification, adapted our provider workforce to new clinical guidance and working conditions, and minimized face-toface contact to protect patients and health care workers.

\section{COVID-19 Laboratory Testing, Ordering, Interfaces, and Communication}

UW Medicine started validating their COVID-19 test in early February and brought their test online on March 2, 2020. The laboratory scaled testing to a capacity of 2,000 tests per day over a 10-day time period.

Laboratory IT had to ensure functional workflows for specimen collection from both traditional sources and from new specimen source locations using provisional collection staff, as some locations had not previously used the EHR (e.g., employee health). We were able to leverage employees' existing EHR records with the guidance of our compliance office by giving employees the option to consent to not separate their employee health data from their patient record. The feasibility of this approach would be dependent on local and state laws.

For other locations which still relied on paper-based and faxed orders (e.g., community screening of nursing homes), workflows to prelog and preprint labels were developed so samples could be accessioned and resulted as quickly as possible upon receipt in the laboratory. New policies guided specimen labeling, and new protocols were developed to ensure safe handling, packaging, and transport of COVID-19 specimens (-Table 2).

Our Laboratory IT group and ITS coordinated to manage diagnostic testing. We first created an unambiguously named COVID-19 order and test component names to integrate into EHRs, predicting that most providers would search for COVID. Severe acute respiratory syndrome (SARS)-CoV-2 was also included as a synonym for the orderable. Of note, for FDA and state validation purposes, the laboratory information system lists SARS-CoV-2 in the name, but this name only appears on printed/faxed reports directly from the laboratory information system.

We decided to make this order not require cosignature, to broaden ability to order. Laboratory information system interfaces to EHRs and to external systems (e.g., public health) were configured.

\section{Clinical Decision Support}

COVID-19 testing and management guidelines are continually evolving. HICS followed these changes and updated institutional policies and protocols. UW Medicine ITS supported clinicians by working closely with HICS and continually updated order sets and documentation templates to ensure that the information was available to clinicians at the proper time in their workflow.

The COVID-19 order set ( - Fig. 3 ) allows direct access to useful hyperlinks, laboratory orders, appropriate International

Table 2 COVID-19 laboratory documents

\begin{tabular}{|l|l|}
\hline Document type & Brief description of document(s) \\
\hline COVID-19 requisition form & Special fillable pdf for clients \\
\hline Self-collected sample information & Client letter for distribution explaining reasons why the sample is not acceptable \\
\hline COVID-19 sample processing policy & Streamlined processes for samples for specimen processing services \\
\hline COVID-19 tiered clients list & Tiered client list for ease of assigning tier in specimen processing services \\
\hline Process for COVID-19 panel requests & Job aid for validation materials, form to track requests for validation samples \\
\hline $\begin{array}{l}\text { Process for sending positive samples } \\
\text { to state health department }\end{array}$ & Job aid for sending samples \\
\hline $\begin{array}{l}\text { Transplant-related protocols } \\
\text { for COVID-19 }\end{array}$ & $\begin{array}{l}\text { Job guideline for donor/recipient testing, labels for application on transplant } \\
\text { samples for easy identification, courier directions for transplant patient samples }\end{array}$ \\
\hline Mitigation plans & Divisional guidelines for pandemic laboratory operations \\
\hline $\begin{array}{l}\text { Policy for nurse draw } \\
\text { on COVID-19 inpatients }\end{array}$ & $\begin{array}{l}\text { Phlebotomy to be done by nursing staff to conserve personal } \\
\text { protective equipment }\end{array}$ \\
\hline Specimen processing volunteer aids & $\begin{array}{l}\text { Intake process for volunteers, description of job duties for volunteers, } \\
\text { self-serve shift sign up worksheet }\end{array}$ \\
\hline Self-attestation for employees & Sign in sheet to attest that employees are symptom-free \\
\hline
\end{tabular}

Abbreviation: COVID-19, novel coronavirus. 
E: SmartSets

$\times$ Remove Pend Sign

COVID-19 Personalize ᄎ

- Reference Information

$\checkmark$ Helpful Links

- UW Medicine COVID-19 Site

- WA DOH COVID-19 Site

- UW Telehealth Job Aids

$\checkmark$ Reason for Visit

$\checkmark$ Reason for Visit

$\square$ Breathing Problem (aka Shortness of Breath)

$\square$ cough

$\square$ Fever

$\square$ Throat Problem (aka Sore Throat)

$\square$ URI

$\checkmark$ Charting

$\checkmark$ Charting

$\square$ covID-19 Note (for both Telehealth and non-Telehealth visits)

$\square$ covID-19 Sample Collection Documentation

$\checkmark$ Orders

$\checkmark$ Labs

$\square$ COVID-19 Coronavirus Qualitative PCR (Normal Status is now being used for ALL visits) Routine, ${ }^{* *}$ DO NOT send in Tube System **

$\square$ Influenza A, B and RSV, Rapid PCR (Strongly consider empiric treatment. To be ordered only if results will change clinical management) (\$\$\$)

Routine

$\square$ Influenza Assay Rapid, Onsite - UWNC only (Strongly consider empiric treatment. To be ordered only if results will change clinical management)

Routine

$\square$ Strep Test Rapid, Onsite (UWNC only)

Routine

$\checkmark$ Diagnosis

$\rightarrow$ Diagnosis

$\square$ cough [R05]

$\square$ Fever, unspecified [R50.9]

$\square$ Shortness of breath [R06.02]

$\square$ Sore throat [J02.9]

$\checkmark$ LOS

- Level of Service Office Visit Click for more

Level of Service Telemedicine (GT modifier | Visit conducted with live, Click for more interactive video \& audio)

Level of Service Telephone (Visit conducted with audio only) Click for more

$\checkmark$ Patient Instructions

$\checkmark$ Patient Instructions

$\square$ patient Instructions for URI and possible COVID-19 testing

$\checkmark$ Ad-hoc Orders

O Search

You can search for an order by typing in the header of this section.

(6) 2020 Epic Systems Corporation. Used with permission

Fig. 3 COVID-19 order set. The UW Medicine COVID-19 order set for our ambulatory electronic health record was built in Epic. We will continue to update this. The latest version can be found at the UW Medicine COVID-19 web site. ${ }^{7}$ (Reprinted with permission of Epic Systems Corporation). 
Classification of Diseases, 10th Revision (ICD-10) diagnoses, billing codes, and discharge instructions. The COVID-19 documentation template ( - supplementary Appendix, available in the online version) includes a checklist of symptoms and risk factors as well as the latest testing recommendations. The template also supports telemedicine and telephonic visits. The reason for visit is also included, since due to conservation of personal protective equipment (PPE), medical assistants now rarely room patients, and thus do not prepopulate this field.

By making quick changes to EHR documentation, such as a single order set, ITS was able to support patient engagement and triage at several critical intersections. One was the nurse triage line that UW Medicine greatly expanded in early March. Using delegated authority and an order protocol, nurses who had never placed orders in the EHR could use a standardized set of laboratory orders and diagnoses without having to learn complex EHR workflows. Another critical improvement was to give front desk staff the ability to access updated appointment templates and cancellation options. This allowed UW Medicine to track COVID-19 related financial impacts.

\section{Results Review}

The COVID-19 test order was integrated into the COVID-19 order set. Results messaging was developed to give ordering providers clinical guidance and to reflect the necessity to err on the side of public health in cases of negative or inconclusive results.

As the major regional referral laboratory for COVID-19, only a fraction of the COVID-19 testing performed was for established UW Medicine patients. The responsibility for communicating results is challenging for patients without an associated UW Medicine primary care provider to perform the follow-up. In response, UW Medicine implemented a centralized results notification process, which uses remote workers instead of front-line clinicians (-Fig. 4). All ambulatory results are routed to a centralized EHR inbox pool staffed by trained professionals. By maintaining a small group of responders, tight and consistent messaging was maintained despite changing guidelines. For patients outside of UW Medicine, laboratory medicine contacted the ordering organizations to communicate positive and inconclusive results.

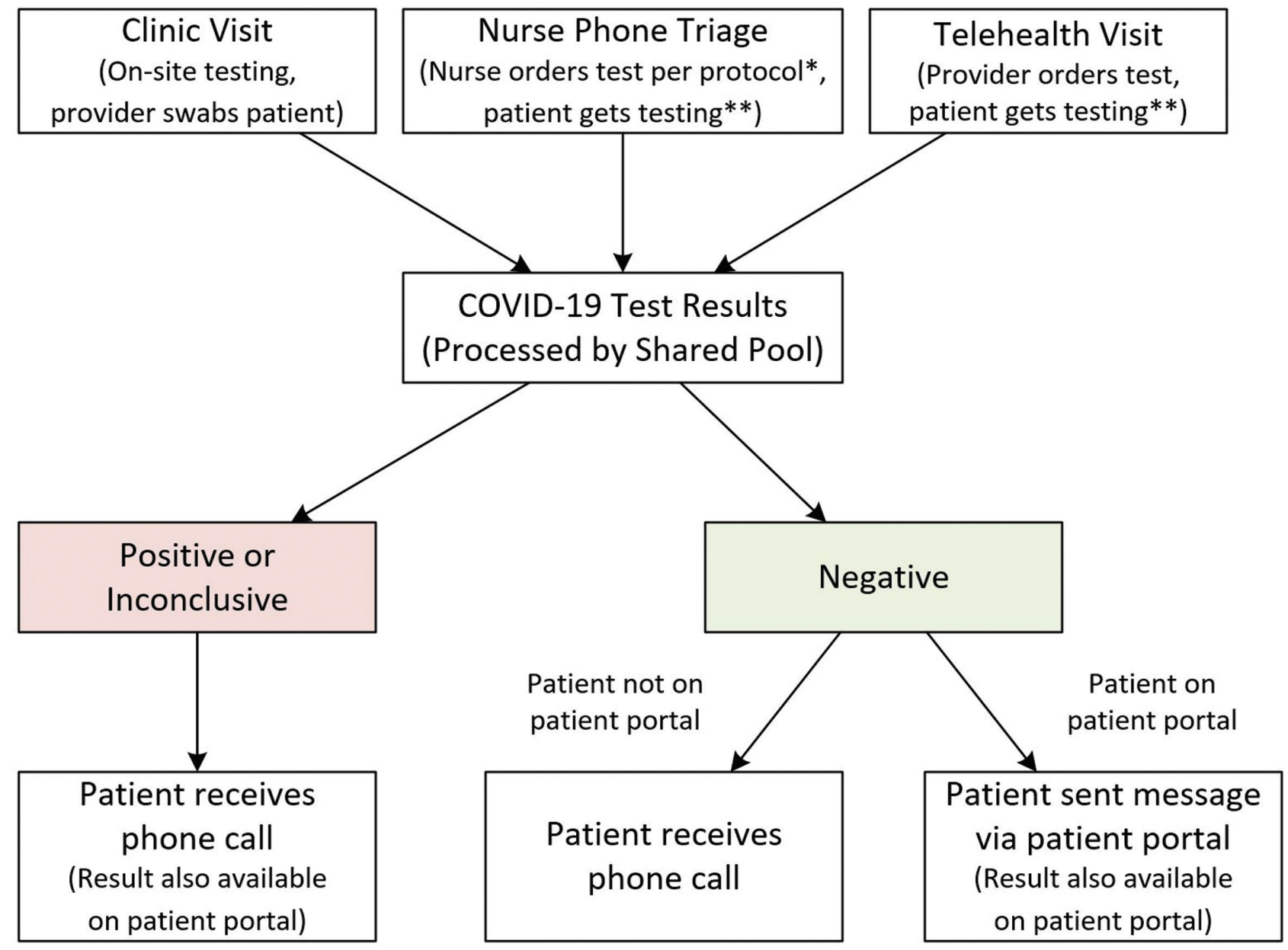

* Only available for COVID-19 testing per protocol

**Patients can get testing from a restricted number of testing sites including drive-through testing

Fig. 4 COVID-19 centralized results notification process. 
Patients with positive COVID-19 results were called, while negative results were available on the patient portal within 1 hour for established UW Medicine patients. A centralized number was provided to patients who missed the call to inform them of the results.

\section{Telehealth for Clinic Visits}

The need to limit exposure of potentially infectious patients and conserve PPE quickly emerged as a critical consideration when faced with a situation of indeterminate length and global supply shortages. This situation caused our practitioners to follow two courses of action: (1) screening patients before they presented in person or (2) screening them remotely while isolating the patient in a room with telemedicine capabilities.

To screen patients for appropriateness of a telehealth visit, UW Medicine aligned our usual screening pathways to match our COVID-19 symptom criteria. Aligned pathways include through scheduling (e.g., appointments through the patient portal, registering for an urgent care appointment online, or calling our contact center to set-up an appointment) and through consultation (e.g., calling our nurse triage line). If patients walked in with respiratory symptoms, they were scheduled for a telehealth visit instead of an in-person evaluation. Language was also added to our web sites and patient portal directing patients with respiratory symptoms to telehealth visits.

UW Medicine moved quickly to provide privileges and train all UW Medicine primary care clinicians in telemedicine. By March 19, approximately 500 providers had been trained and received privileges, including close to $100 \%$ of our primary care doctors. UW Medicine expanded COVID-19 telemedicine to clinics who care for vulnerable patients such as oncology, transplant, cardiopulmonary, and end-stage renal patients. Providers were able to access telemedicine training videos, ${ }^{8}$ job aids, and other resources from the UW Medicine intranet. The telemedicine e-learning at UW Medicine is comparable to that offered by the Washington Telehealth Collaborative. ${ }^{9}$ Additional learning topics included signing up for telemedicine, billing documentation, and how to use the EHR in conjunction with the teleconferencing tool are provided to UW Medicine providers on an internal intranet page.

ITS helped to acquire equipment, managed software licenses, supported remote access, trained practitioners, and integrated teleconferencing into our ambulatory EHR. This ensured that the UW Medicine systems could handle the influx of users and increased utilization of our network and resources. We engaged UW Medicine compliance in helping with processes to address billing and privacy related concerns. We worked with clinical leaders to establish a consistent methodology to educate patients on how to connect to telemedicine appointments.

We determined the best tools and workflows to mask the phone numbers of personal devices used with those of the designated clinic or the UW Medicine contact center. To change the caller ID to instead display the phone number of a designated clinic or the UW Medicine contact center, providers were instructed to use either the Doximity Dialer application or use a UW Medicine call forwarding feature through our phone system (Avaya/Asterisk). Users could call a designated phone number, enter a numeric password, and proceed to dial the patient's phone number. This allowed more practitioners to work remotely.

\section{Telehealth in Intensive Care Units}

UW Medicine moved quickly to launch telemedicine capability in the ICUs, to allow intensivists to engage with patients via tele-ICU carts, rolling devices with two high definition cameras and a screen on a pole with wheels. ${ }^{10}$ Tele-ICU capability offered a high degree of flexibility, as the tele-ICU carts were rapidly deployed at three of our four hospitals, allowing UW Medicine to surge capacities of specialists virtually across our different institutions as needed. The tele-ICU carts allowed practitioners to conduct bedside visits, eliminated the time for donning, and doffing PPE, and conserved PPE.

\section{Surge Planning}

As COVID-19 spreads, ITS has been involved in surge planning. This includes planning for expedited procedures for granting emergency-level access to systems, changes to the EHRs, and other systems to allow tracking of patients, who may be placed in nonclinical areas, and a process to rapidly extend our network for new clinics and treatment areas.

UW Medicine is planning to convert ambulatory clinic space at the hospitals to inpatient COVID-19 assessment areas. This requires the same level of effort and scrutiny of EHR build effort as establishing a new clinic. The inpatient tracking requirements are much stricter than in ambulatory settings and are critical for assessing who may have encountered presumed or confirmed positive patients. We are working on securing more equipment (desktop computers) to support the IT infrastructure of these surge locations.

ITS also supported mobile testing clinics such as the nation's first drive-through testing site. This meant extending network connectivity and providing systems to support collecting specimens from patients with unknown COVID-19 status while in their cars. Initially, UW Medicine leveraged cellular hotspots normally used for clinics during a power outage and while this worked as a short-term solution, ITS quickly implemented microwave line of sight systems that provided more robust connectivity.

\section{Workforce Issues}

ITS leadership recognized the need to quickly leverage telework to keep the workforce healthy, stable, and productive through the pandemic. In early March, ITS pivoted to teleworking for all personnel who did not need to be physically present to perform their job duties. The enterprise already leveraged teleconferencing tools due to UW Medicine's geographic spread and could focus on increasing bandwidth and access to the virtual private network (VPN), teleconferencing tools, and file-sharing resources.

HR quickly released policies and guidance on how to manage a teleworking workforce. Staff checked out their desktop hardware such as monitors, keyboards, and mice. 
Remote staff were expected to be available via phone, e-mail, and instant messaging.

\section{Security}

Security and compliance quickly emerged as focus areas, given the move to teleworking and the rise in attacks by malicious actors during the pandemic.

The speed of the pivot to social distancing coupled with supply chain constraints, meant that many ITS staff used personal devices while teleworking when not handling protected health information. Physicians were able to securely access the EHR via vendor applications. And while ITS was able to surge bandwidth for the system, staff faced local bandwidth constraints from residential internet providers. This placed a unique strain on maintaining security, as the protections offered via our secure VPN were only accessible if staffs logged in to the VPN, an extra step that many in the newly teleworking workforce were unfamiliar with. The technical team pushed out guidance via ITS leadership e-mails and UW Medicine compliance and security worked together to rapidly update, consolidate, and disseminate teleworking guidance.

The COVID-19 pandemic presented a security threat to systems. While UW Medicine regularly faces and fends off cyber-attacks, security saw a rise in phishing and attempts to use COVID-19 to lure health care workers to web sites laced with malware. The security team collaborated with local and regional security partners, including the Federal Bureau of Investigation (FBI), to monitor the health of the UW Medicine network and devices. ITS plans continued workforce education on good security hygiene, identify procedures for investigating attacks on home computers of teleworking staffs, identifying methods for performing forensics on home computers, and disseminating security software for mobile devices.

\section{Lessons Learned}

UW Medicine pivoted quickly to address clinical needs as they arose with COVID-19. There are three areas that UW Medicine would have done things differently, given the benefit of hindsight. First, we would have prioritized the full integration of our video conferencing solution into the ambulatory EHR. Second, we would have placed more emphasis on broadly expanding our telemedicine solution. While we had a foundation to build from given our existing program, we would have benefitted from having more providers trained and more cameras in place. And finally, we would have had a larger supply of equipment on hand to support both teleworking and telemedicine.

\section{Next Steps}

IT is a cornerstone of organizational response to coordinating operational and clinical needs and must rapidly rearrange infrastructure, policies, and priorities to remain responsive. At UW Medicine, we anticipate expanding and adapting our response to COVID-19 by using the framework enacted in our initial strategy.

ITS is working with regional and national partners to build solutions that allow organizations to share information

Table 3 Top 10 things your health care IT organization can do now

These are the top 10 actions you can take now to help your organization prepare for COVID-19 or future infectious disease emergency scenarios.

1. Establish your new or evaluate your existing IT response structure. Be sure that points of contact and processes will work for this situation. Plan for the long haul. You will need IT services to surge support for weeks or months. Ensure you have a deep bench of experts in key areas to sustain the demand.

2. All updates to your EHR must be evaluated and centrally disseminated as quickly as possible. Your IT personnel must be able to do this around the clock. Ensure that your information security team has a rapid process to assess, document, and approve risk decisions and exceptions during the emergency.

3. Quickly prepare multiple sites with telehealth capability. This will allow patients and practitioners to flow between different sites. Begin training your practitioners immediately.

4. Assess remote user capability, licenses, software, hardware and bandwidth limitations to connect to your internal systems to ensure your systems can handle the influx of users and increased utilization of your network and resources.

5. Assess how your organization can be nimble with granting access to systems and sites in emergencies. Start planning now for emergency-level access that allows people to surge and flow between sites.

6. Make patient screening tools accessible prior to presenting. Priority needs to be on ensuring your patients know how to self-screen.

7. Establish a centralized intranet site for disaster management and communication. This includes an incident command dashboard of automated metrics to help assess the evolving situation.

8. Identify the role of IT in sending communication to the workforce. Test dissemination methods to ensure they reach your entire workforce. Review communication distribution lists to ensure that they accurately reflect the internal, external, partner, and other groups that are critical to your response.

9. Prepare for increased help desk support requirements, and ensure your staff are prepared to answer questions. Quickly resolve issues with clinicians using new telehealth capabilities and newly teleworking employees.

10. Plan for large scale remote work. This will require workforce provisioning of equipment and policies and procedures for managing a remote workforce.

Abbreviations: EHR, electronic health record; IT, information technology. 
about open beds, capacity, and current stock of key items such as masks and ventilators. This work may support the use of predictive analytics to forecast patients at different levels of acuity, which will allow for a calculated response and the ability to divert patients to different hospitals in the region based on clinical presentation and available resources.

Timeliness of communication between patients and the health care team is critical. This can be accomplished through increased enrollment in web portals and transitioning to patient text-based messaging and telemedicine visits. Increasing portal access may improve the ability to exchange medical records with additional care providers for patients diverted to other facilities as the result of matching patients to community providers.

There are additional opportunities to engage more providers should the epidemic continue for a longer period. Key considerations include streamlining onboarding and decreased training time, improving organizational security, providing health care workers with information about phishing and other attacks, and providing resources to report them.

\section{Conclusion}

The unfolding pandemic of COVID-19 necessitated a previously uncharted reorganization of health care system infrastructure, processes, and priorities. Surge capacity planning, patient triage, infection control, supply chain management, communication, and online patient management were identified as key challenges in the recent experience of health systems in Asia. ${ }^{11,12}$ UW Medicine leveraged established HICS command methodologies to rally the resources of our organization in response to this unprecedented event. Through a shared sense of purpose and nimble response to clinical and operational requests, the ITS team played an integral role in responding to this public health emergency. Some recommendations for other organizations to consider are in -Table 3. More detailed, regularly updated information can be found on our external website. ${ }^{7}$

\section{Clinical Relevance Statement}

This paper provides lessons learned and guidance for hospital IT leaders to consider while positioning their organizations to support clinical needs for the COVID-19 pandemic response.

\section{Multiple Choice Questions}

1. An effective metric to determine the burden of COVID-19 on the health system might be

a. Number of COVID-19 tests by facility per day

b. Laboratory turnaround time

c. Outpatient no shows per day

d. Emergency department length of stay

Correct Answer: The correct answer is option a. The number of COVID-19 tests by facility per day is the most direct measure of the burden of COVID-19 on the health system, although each of the metrics listed may be affected by COVID-19 burden. Testing reflects the number of patients being seen throughout the health system (and the region in the case of regional laboratories) with a clinical suspicion for COVID-19. The other metrics all have the potential to be impacted by factors unrelated to COVID-19 burden.

2. COVID-19 disease is caused by
a. $229 \mathrm{E}$ ( $\alpha$ coronavirus)
b. MERS-CoV
c. SARS-CoV-2
d. HKU1 ( $\beta$ coronavirus)

Correct Answer: The correct answer is option c. COVID19 (coronavirus identified in 2019) is caused by SARSCoV-2 (the second identified severe acute respiratory syndrome associated with coronavirus), to distinguish it from the SARS epidemic caused by SARS-CoV- 1 identified in 2002. There are many other coronavirus isolates that cause human disease, notably, MERS (Middle Eastern Respiratory Syndrome identified in 2012), and the common seasonal coronavirus types 229E ( $\alpha$ CoV) and HKU1 $(\beta \mathrm{CoV})$.

3. Close contact to a patient with COVID-19 is contact within approximately this distance for a prolonged amount of time
a. $1 \mathrm{~m}$
b. $2 \mathrm{~m}$
c. $3 \mathrm{~m}$
d. $5 \mathrm{~m}$

Correct Answer: The correct answer is option b. The CDC identifies close contact as "being within approximately 6 feet $(2 \mathrm{~m}$ ) of a COVID-19 case for a prolonged period of time" (https://www.cdc.gov/coronavirus/2019-ncov/php/ risk-assessment.html, accessed March 28, 2020).

Note

A previous version of this work is available on the UW Medicine COVID-19 web site at https://covid-19.uwmedicine.org/. In the interest to publish this paper while the COVID-19 pandemic is still ongoing to share lessons learned with others as quickly as possible, this paper has undergone an abbreviated and unblinded peer review. Users are advised to use the recommendations in this paper in a judicious manner.

Protection of Human and Animal Subjects

We reviewed this work with our Human Subjects Division. They concluded it was not human subject research.

Funding

None. 


\section{Conflict of Interest}

M.G.L. reports his FTE is partly paid by UW Medicine IT Services.

\section{Acknowledgment}

We would like to acknowledge UW Medicine IT Services staff for their assistance in implementing the interventions described, and for their support when collecting the information for this manuscript. We like to thank Cris V. Ewell, Stephanie Klainer, Sean D. Mooney, and Adam Wilcox for their critical review of the manuscript. We would also like to thank Beth Britt, Anne M. Cent, Mary P. Horan, Staci Garrison, Anna Marti, Adam Parcher, Thomas Payne, John Scott, and Kristine Wegener for their contributions, and R. Max Vrooman, Joe Pankiewicz, and Susan Onstad for their ongoing work on the UW Medicine Incident Command Dashboard.

\section{References}

1 Bhadelia N. Coronavirus: hospitals must learn from past pandemics. Nature 2020;578(7794):193

2 Holshue ML, DeBolt C, Lindquist S, et al; Washington State 2019-nCoV Case Investigation Team. First case of 2019 novel coronavirus in the United States. N Engl J Med 2020;382(10): 929-936

3 Centers for Disease Control and Prevention. Coronavirus disease 2019 (COVID-19) cases in the U.S. Available at: http://www. cdc.gov/coronavirus/2019-ncov/cases-updates/cases-in-us.html. Accessed March 17, 2020

4 Persoff J, Ornoff D, Little C. The role of hospital medicine in emergency preparedness: a framework for hospitalist leadership in disaster preparedness, response, and recovery. J Hosp Med 2018;13(10):713-718

5 Chacko S, Randolph R, Morsch G. Disaster medicine: public health preparedness for natural disasters. FP Essent 2019; 487:17-22

6 Bryson-Cahn C, Duchin J, Makarewicz VA, et al. A Novel Approach for a Novel Pathogen: using a home assessment team to evaluate patients for 2019 novel coronavirus (SARS-CoV-2). Clin Infect Dis 2020. Doi: $10.1093 /$ cid/ciaa256

7 UW Medicine. COVID-19 resource site. Available at: https://covid19.uwmedicine.org/Pages/default.aspx. Accessed March 22, 2020

8 Washington State Hospital Association. WA Collaborative Health Care Professional Telehealth Training_Jan2019_final_video. Available at: https://vimeo.com/344187400. Accessed March 23, 2020

9 Washington State Hospital Association. Washington State Telehealth Collaborative. Available at: https://www.wsha.org/policyadvocacy/issues/telemedicine/washington-state-telemedicinecollaborative/. Accessed March 22, 2020

10 Teladoc Health. InTouch Lite. Available at: https://intouchhealth. com/telehealth-devices/intouch-lite-and-lite-with-boom/. Accessed March 23, 2020

11 Cao Y, Li Q, Chen J, et al. Hospital emergency management plan during the COVID-19 epidemic. Acad Emerg Med 2020. Doi: 10.1111/acem.13951

12 Liew MF, Siow WT, MacLaren G, See KC. Preparing for COVID-19: early experience from an intensive care unit in Singapore. Crit Care 2020;24(01):83 